\title{
Immediate detection of irrigant absorption during transurethral prostatectomy: case report
}

\begin{abstract}
A transurethral prostatic resection is described in which immediate detection of a rapid massive irrigant absorption could be made by the use of ethanol-lagged irrigating fluid and repeated measurements of the ethanol concentration in the patient's expired breath. This monitoring enabled the surgeon to prevent further absorption by concluding the operation before symproms resulted. Furosemide was given immediately to promote renal excretion of the absorbed irrigant, and the intravenous infusions were temporarily restricted to limit the intravascular fluid load. In retrospect, volumetric measurement showed that a total of $2410 \mathrm{ml}$ of irrigant had been absorbed.
\end{abstract}

Water intoxication from the absorption of irrigating fluid is a potential hazard associated with transurethral prostatic resection. ${ }^{1-5}$ In routine resections the degree of irrigant absorption is usually assessed from the serum sodium concentration measured at the end of the resection or from the volumetric irrigating fluid balance, while isotopic methods may be used for research purposes. ${ }^{2,3}$ Recently a simple non-invasive method to detect irrigant absorption, relying on ethanol tagging of the irrigating fluid, was described. In a study of 50 resections the presence and volume of irrigant absorbed could be indicated immediately from measurement of the ethanol content of the expired breath. ${ }^{4}$ Here we report a case where ethanol monitoring of the irrigant absorption facilitated the early instigation of treatment and of steps to prevent further absorption, thereby contributing to the ultimately successful outcome.

\section{Key words}

SURGERY: transurethral resection of the prostate; COMPLICATIONS: Bleeding, irrigant absorption, hyponatraemia; ALCOHOL.

From The Department of Anaesthesiology, Huddinge University Hospital, S-141 86 Huddinge, Sweden.

Address correspondence to: Dr. R.G. Hahn.

\section{Case report}

The patient was a 70 -year-old man weighing $83 \mathrm{~kg}$, with a history of mild angina pectoris and cardiac insufficiency. Epidural anaesthesia was given. An intermittent bladderfilling technique was used during the resection, the irrigating fluid being 1.5 per cent glycine +1 per cent ethanol (Travenol, Sweden). Postoperatively, this technique was continued using 0.9 per cent saline. The ethanol concentration in the expired breath was measured repeatedly during surgery with an Alcolmeter SD-2 (Lions Ltd, Barry, Wales). Peroperative venous blood sampling was also carried out repeatedly for determination of the serum sodium concentration by flame photometry. ${ }^{5}$ A volumetric fluid balance was obtained every 5 or $10 \mathrm{~min}$ throughout resection by changing the irrigating fluid bag and collection bucket. The blood content of each collection bucket was measured by a photometer (HemoCaps $^{(1)}$ system, Leo, Helsingborg, Sweden), using the blood haemoglobin concentration for reference, and the volume of irrigant absorbed was calculated as the difference between the volumetric fluid balance and the blood loss. ${ }^{\mathrm{s}}$

The course of the transurethral prostatic resection is summarized in the Figure. During the operation $51 \mathrm{~g}$ of benign prostatic tissue was removed. Sixty minutes after the start of the resection the ethanol monitoring indicated that irrigant was being absorbed at a high rate. The surgeon was informed, and the operation was terminated. To promote renal excretion of the absorbed fluid $40 \mathrm{mg}$ of furosemide was immediately given intravenously. The total blood loss during the surgical procedure was $1510 \mathrm{ml}$ and the volume of irrigant absorbed, as indicated by the volumetric fluid balance, was $2410 \mathrm{ml}$. The blood ammonia concentration was normal.

In the postoperative ward the bleeding continued and packed red cells and plasma were transfused. In spite of vigorous bladder irrigation, a complete bladder outlet obstruction developed. The patient was returned to the operating theatre for evacuation of blood clots, and repeated attempts to coagulate the open vessels in the 


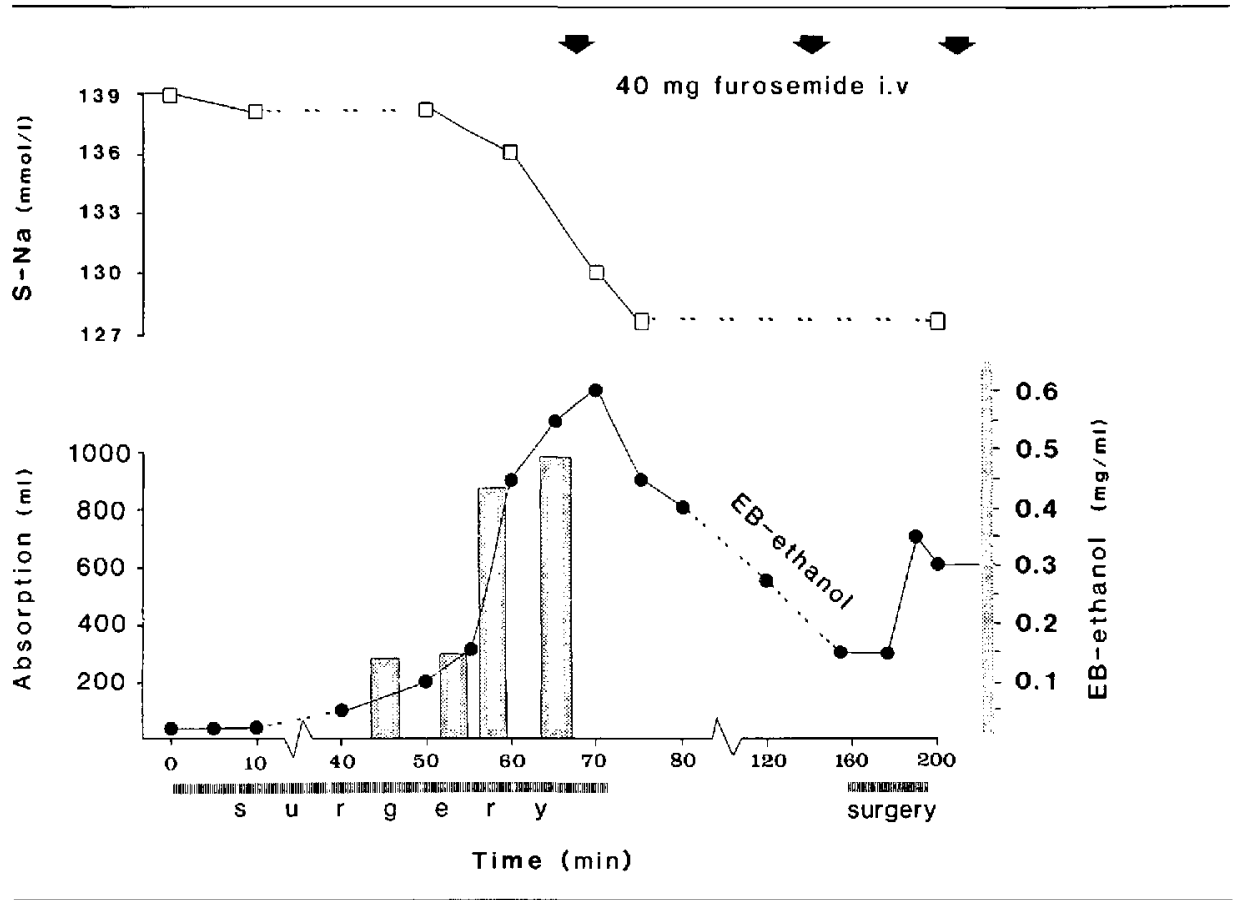

FIGURE Schematic presentation of the irrigant absorption as assessed by three different methods in a patient undergoing transurethral prostatectomy. The serum sodium concentration (S-Na) was measured repeatedly during the course of resection. Secondly, the volume of irrigant absorbed was calculated as the difference between the irrigating fluid input and output with correction for the amount of blood lost (bars; not cumulative values). Lastly, the ethanol concentration in the expired breath (EB-ethanol; expressed as the corresponding blood ethanol concentration in $\mathrm{mg} \mathrm{ml}^{-1}$ ) was measured at $5-10 \mathrm{~min}$ intervals; the irrigant was tagged with ethanol and therefore this determination indicared irtigating fluid absorption. Some measurements from the reoperation was also presented (here ethanol-tagged irrigating fluid was only used towards the end). Arrows on top indicate the timing of the intravenous injections of furosemide.

prostatic fossa. Ethanol monitoring indicated that irrigant was again being absorbed at a high rate (Figure). During both the operation and the re-operation there were no adverse effects from the irrigating fluid or from the ethanol. The circulatory status was stable. A laparatomy was performed on the following day due to persistent bleeding, but thereafter recovery was uneventful and the patient was discharged from the hospital nine days later.

\section{Discussion}

Three methods were used to follow the irrigating fluid absorption during the resection in this patient. The first method employed the change in the serum sodium concentration as an indication of the degree of electrolyte dilution produced by the absorbed irrigant. ${ }^{3}$ The second method consisted of volumetric measurement, which uses the difference between irrigating fluid input and output with correction for the amount of blood lost as a measure of the volume of irrigant absorbed. ${ }^{5}$ The third method employed expired-breath tests in the detection of irrigant absocption. It has been shown that, when irrigating fluid is tagged with ethanol, the ethanol content of the exhaled breath correlates closely with both the volumetric measurement of the absorption and the dilution of serum sodium. ${ }^{4}$ In this patient, all of these methods indicated rapid massive absorption at about the same time (Figure). However, in our opinion, ethanol indication is the most convenient method of monitoring irrigant absorption; a single expiration of the patient into the mouthpiece of an Alcolmeter will give an estimate of the irrigant absorption from the ethanol concentration shown on the display. ${ }^{4} \mathrm{An}$ additional advantage over measurement of the serum sodium concentration and of the volumetric fluid balance is that the results relating to absorption are available immediate- 
ly. In the reported patient, this allowed us to prevent untoward effects of the irrigant: furosemide was given in immediate conjunction with the absorption to induce diuresis, and the acute intravascular overhydration from the irrigating fluid was limited by withholding the transfusion of packed red cells and plasma necessitated by the peroperative blood loss until after completion of resection. Furthermore, and most important, the operation could be terminated when irrigant was being absorbed at the rapid rate of $1000 \mathrm{ml} \cdot 10 \mathrm{~min}^{-1}$ as measured volumetrically (Figure); this intervention clearly saved the patient from absorbing an additional (and possibly substantial) load of irrigating fluid. With this regimen, the patient remained comfortable and asymptomatic.

\section{References}

1 Desmond J. Complications of transurethral prostatic surgery. Can Anaesth Soc J 1970; 17: 25-36.

2 Madsen P, Kuni H, Naber $K$. Various methods of determining irrigating fluid absorption during transurethral resection of the prostate. Urol Res $1973 ; 1: 70-8$.

3 Hahn $R$. Relations between irrigant absorption rate and hyponatraemia during transurethral resection of the prostate. Acta Anaesthesiol Scand 1988; 32: 53-60.

4 Hahn $R$. Ethanal monitoring of irrigating fluid absorption in transurethral prostatic surgery. Anesthesiology 1988; 68: 867-73.

5 Hahn R. Influence of variations in blood haemoglobin concentration on the calculation of blood loss and volumetric fluid balance during transurethral resection of the prostate. Br J Anaesth 1987; 59; 1223-9.

\section{Résumé}

L'utilisation d'une solution d'irrigation marquée d'éthanol et des mesures répétées des concentrations de l'éthanol expiré chez un patient devant subir une résection transurétrale de la prostate est décrite afin de permettre une détection immédiate d' une absorption massive du liquide d' irrigation. Cette surveillance a permis au chirurgien de prévenir une plus grande absorption. Du furosémide a été administré immédiatement afin de faciliter l' excrétion rénale du liquide absorbé en même temps qu'une restriction de l'apport hydrique afin de limiter la surcharge. Rétrospectivement on a calculé que $2410 \mathrm{ml}$ de liquide d'irrigation a été absorbé. 